\title{
Optical modelling of a si-based DBR laser source using a nanocrystal Si-sensitized Er-doped silica rib waveguide in the C-band
}

\section{Ciminelli \\ c.ciminelli@poliba.it}

\section{P. Frascella}

Dipartimento di Elettrotecnica ed Elettronica, Politecnico di Bari, Via Re David 200, Bari, Italy

UCC Department of Physics, University College Cork, Lee Maltings, Prospect Row Cork, Ireland

\section{N. Armenise}

The availability of reliable silicon-based laser sources is at the basis of the integration of photonic and microelectronic devices on a single chip with consequent development of wavelength division multiplexing telecommunication systems.

A high efficiency Si-based laser source with good stability at room temperature would encourage and push the large scale of integration of electronic and photonic devices within a single chip.

Several techniques have been proposed for generating light with an internal quantum efficiency some order of magnitude greater than that typical of silicon $\left(10^{-6}\right)$ by using either electrical or optical pumping. Among them we mention the improvement of some fabrication process steps, reduction of the channels of non-radiative recombination, quantum confinement, the use of silicon nanocrystals (Si-ncs) incorporated in a silica matrix. This last technique is used in combination with $\mathrm{Er}^{3+}$ doping to generate light emission around $1500 \mathrm{~nm}$ in silicon, since Er-doped Si-ncs behave as electron-hole pairs trap, and the presence of Er shifts the emission peak to around $1500 \mathrm{~nm}$.

In this paper we have pointed out the optical model of a Si-based DBR laser including a Si-ncs Er-doped $\mathrm{SiO}_{2}$ rib waveguide, working at a wavelength in C-band. In particular, after a brief description of the structural and optical properties of the silicon crystals, we report on the model and design of the Er:Si-nc/SiO 2 rib waveguide, of the optical cavity and of the Bragg mirrors. [D0I: 10.2971/jeos.2008.08017]

Keywords: laser, erbium doping, silicon nanocrystal

\section{INTRODUCTION}

The integration of photonics and microelectronics devices on a single chip and further development of wavelength division multiplexing communication systems, depend on the availability of both reliable optical amplifiers and silicon-based laser sources. As for the optical amplifiers, except for the semiconductor optical amplifiers, their fundamental role is the conversion of the pump photon energy into a signal photon energy. Unfortunately, all the rare-earth doped or Raman amplifiers have to use pump lasers, which, of course, contribute to arise the final amplifier cost. Moreover, in Er-doped fibre amplifiers, for any pumping scheme, i.e. forward, backward, bidirectional or reflection pumping, both the optical signal and pump waves are co/counter propagating in the same waveguide. This is not an issue for optical fibre amplifiers, but it represents a serious problem in terms of design flexibility and device functionality when the amplifier must be integrated in photonic integrated circuits.

A high efficiency Si-based laser source working at room temperature would promote and push large scale integration of electronic and photonic devices within a single chip. This is an actual problem mainly related to the indirect band gap characteristics of the bulk silicon, which means a light emission assisted by a phonon. Thus leading to an emission rate less than the non-radiative decay rate, and, therefore, to a low internal quantum efficiency of the order of $10^{-6}$.
Several techniques have been proposed and experimented for producing light emission with a reasonable efficiency in silicon by using either electrical or optical pumping.

In [1] the non-radiative recombination rate has been reduced by improving some technological parameters, e.g. small area metal pads, thin doped regions in high quality Si substrates, and texturing of Si surface. The electroluminescence has been proved to be $1 \%$. In [2] the channels of non-radiative recombination are significantly reduced by utilizing strains produced by some defects dislocation. Also in this case an efficiency of $1 \%$ was obtained. Er-doped silicon waveguides have been used to demonstrate near infrared $(1.54 \mu \mathrm{m})$ emission [3]. However, a strong reduction of the light emission efficiency has been observed at room temperature. Quantum confinement represents another approach to the improvement of the emission efficiency in Si structures. Good luminescence has been demonstrated in the visible spectrum for the porous silicon [4], even if with low reproducibility and low reliability due to the high reactivity of the sponge structure [5].

However, once demonstrated that the spatial carrier confinement induces an increase of the light emission efficiency, a technique based on the growth of silicon nanocrystals (Si-nc) embedded in $\mathrm{SiO}_{2}$ matrix, has been experimented [6] with the further advantage to utilize electrical pumping. 
Optical gain in Si-ncs has been proved in [7], where a luminescence peak around $800 \mathrm{~nm}$ and a good stability mainly due to a good quality $\mathrm{Si} / \mathrm{SiO}_{2}$ interface have been claimed for Si-ncs with a diameter of $3 \mathrm{~nm}$ and a concentration of $2 \times 10^{19} / \mathrm{cm}^{3}$. Si-ncs are excited by hot electrons tunnelling in the region where ncs are. Total efficiency of the device increases with the density of Si-ncs. To obtain light emission in the near infrared, around $1.5 \mu \mathrm{m}$, with an efficiency of about $1 \%$, Si-ncs have been Er-doped [8] or Ge/Si quantum dots have been used [9].

Although silicon photonics is the main scientific interest of many research groups all over the world, mostly for the integration compatibility with electronic integrated circuits and micro electromechanical systems, much effort must be spent to improve the lifetime and reliability of active devices. An additional disadvantage for silicon laser sources is low modulation speed $(\sim 1 \mathrm{MHz})$, which implies the use of external modulators in high transmission rate communication systems.

However, nonlinear effect in Si structures, together with the potential of modifying the silicon properties by incorporating nanostructures, show two possible ways to realize Si coherent light sources, i.e. two possible ways for achieving gain and, then, light emission in Si structures.

The first one makes use of a system consisting of Si ncs in host silica matrix, doped by erbium ions. When the optical pumping occurs, photons are absorbed by the Si ncs. Electron-hole pairs are generated within the nanocrystals and thus form confined excitons via interband transitions. Two main radiative processes can be identified. The first one is the stimulated emission of erbium ions at $1540 \mathrm{~nm}(\sim 0.8 \mathrm{eV})$ corresponding to the relaxation of $\mathrm{Er}^{3+}$ from the excited state $\mathrm{I}_{13 / 2}$ to $\mathrm{I}_{15 / 2}$. The indirect pumping of the Erbium ions by means of Si ncs is achieved by a non radiative energy transfer $[10,11]$. The second radiative process that can be considered is the radiative recombination of excitons within Si-ncs, inducing a photoluminescence at $\sim 1.6 \mathrm{eV}$. The presence of Er ions strongly reduces the luminescence contribution at $\sim 1.6 \mathrm{eV}$ and enhances the component at $\sim 0.8 \mathrm{eV}$. The spatial confinement induces an increase of the radiative recombination rate. In addition, the Er-doping shifts the emission peak from about 800 $\mathrm{nm}$ to around $1500 \mathrm{~nm}$. Non radiative processes, such as the upconversion and the inter Si-ncs energy transfers, reduce the quantum efficiency of the erbium photoluminescence.

The second effect that can be used for achieving gain in silicon material is the Raman effect: optical pumping at $\omega_{p}$ in an optical cavity made of silicon amplifies the initial lattice vibrations (Stokes radiation at $\omega_{S}$ ) with a very high gain. Due to Raman effect, the frequency of the optical pump experiences a partial shift caused by the atoms vibrations; the amplification of the signal at $\omega_{s}$ is converted in a continuous generation by means of the resonant cavity [12].

In this paper we present the optical modelling of a Si-based DBR laser including a Si-ncs sensitized Er-doped $\mathrm{SiO}_{2}$ rib waveguide, to be used in telecommunication systems at operating wavelengths in the C-band. In particular, after a brief description of the structure and of the optical properties of the silicon nanocrystals, we report on the modelling and de- sign of the Er:Si-nc/SiO ${ }_{2}$ rib waveguide, of the optical cavity and of the Bragg gratings. Two different numerical methods have been used for modelling the waveguide, i.e. the effective index method and the finite element method.

\section{STRUCTURAL AND OPTICAL PROPERTIES OF Si-nC}

\subsection{Recombination processes in Si}

Since silicon has an indirect band-gap, radiative recombination requires one phonon, thus reducing significantly both the probability and rate of radiative recombination.

The lifetime $\tau_{r}$ of the hole-electron pair, giving rise to a radiative recombination, is about $10 \mathrm{~ms}$ for a temperature $>20 \mathrm{~K}$ $[13,14]$, while in direct band semiconductors $\tau_{r}$ is of the order of $100 \mathrm{~ns} . \tau_{r}$ is given by

$$
\tau_{r}=\frac{1}{B\left(n_{0}+\Delta n\right)},
$$

where $B$ is the coefficient of radiative recombination, $n_{0}$ is the density of doping and $\Delta n$ the density of the injected carriers.

One should also consider that here exist other non radiative recombination processes, such as the Shockley-Hall-Reed (SHR) recombination, due to traps related to defects or impurities in the crystal $[15,16]$. This last process is characterized by a lifetime of minority carriers at low injection levels given by

$$
\tau_{S H R}=\frac{1}{N_{T} v_{t h} \sigma_{T}},
$$

where $N_{T}$ is the trap density, $v_{t h}$ the thermal velocity of minority carriers, and $\sigma_{T}$ the cross section for minority carriers. The SHR recombination lifetime is about $100 \mu \mathrm{s} \ll \tau_{r}$ at room temperature. This justifies the probability of non radiative recombination higher than the radiative one. Also Auger recombination, that is a very fast non radiative process [17], occurs in doped Si n-or-p-type. The lifetime $\tau_{\text {Auger }}$ of a hole-electron pair for an Auger recombination is around $10 \mu \mathrm{s}$ and is expressed as

$$
\tau_{\text {Auger }}=\frac{1}{C_{p} p_{0}^{2}} \quad \text { or } \quad \tau_{\text {Auger }}=\frac{1}{C_{n} n_{0}^{2}},
$$

with $C_{p}$ and $C_{n}$ Auger coefficients ehh ed eeh, respectively and $p_{0}$ and $n_{0}$ are the densities of doping impurities.

The total lifetime is

$$
\frac{1}{\tau}=\frac{1}{\tau_{r}}+\frac{1}{\tau_{S H R}}+\frac{1}{\tau_{\text {Auger }}} .
$$

Silicon does not emit easily light (the efficiency is as low as $10^{-6}-10^{-7}$ ) because non radiative processes are faster than the radiative ones, thus one should reduce the radiative lifetime or increase the non radiative lifetime to improve the quantum efficiency.

\subsection{Si nanocrystals}

Since the non radiative recombination phenomena are dominant with respect to the radiative ones, crystalline silicon is 
unsuitable for realizing optoelectronic active devices. To improve quantum efficiency one should limit the movement of an exciton within a restricted space to reduce the probability of occurrence of non radiative recombination phenomena. Simultaneously, by confining the exciton in a volume of the order of a few $\mathrm{nm}^{3}$ the quantum efficiency is increased because of the increase of radiative recombination rate.

At this size, the matter show new properties. In fact, when the size of the confinement region is comparable with the electron and hole De Broglie's wavelength, the particles cannot move freely and quantum confinement effects arise, as it occurs in nanostructures, e.g. Si-ncs in a $\mathrm{SiO}_{2}$ matrix.

Depending on the shape of nanostructure, quantum confinement can occur in one direction, two directions or three directions in the space. Quantum confinement effect in any confinement direction induces a change of the wave function and discrete energy levels are generated.

Si-ncs are very complex material systems and several attempts have been made to pointing out a model of their optical properties and band structure. However, numerical and experimental results are in general not in good agreement. What has been so far confirmed by both theoretical and experimental works is that the effective band-gap of Si-ncs increases when their size decreases down to $10 \mathrm{~nm}$. In particular, the quantum confinement effect occurs when Si-nc size is less than the Bohr radius of the exciton $(=4.3 \mathrm{~nm}$ for Si bulk).

\subsection{Photoluminescence of $\mathrm{Si} \mathrm{nc}$}

Si-ncs can be grown in a $\mathrm{SiO}_{2}$ matrix by using PECVD (Plasma Enhanced Chemical Vapour Deposition) to deposit $\mathrm{SiO}_{x}(x<$ 2) film and high temperature annealing $\left(1000-1250^{\circ} \mathrm{C}\right)$ to separate $\mathrm{Si}$ and $\mathrm{SiO}_{2}$ phases from the initial $\mathrm{SiO}_{x}$ [18].

When Si-ncs are pumped by an Ar-Kr laser photoluminescence, measurements can be carried out and experimental results have confirmed the quantum confinement of carriers, because a progressive redshift of the peak of photoluminescence can be observed due to the decrease of ncs size (the band-gap becomes wider causing the redshift).

A not negligible difference between numerical and experimental results in the band-gap evaluation has been found. An attempt to explain this effect has been proposed in relation with the presence of the oxygen that creates double bonds $\mathrm{Si}=\mathrm{O}$, traps electrons and forms localized states in the bandgap of ncs with diameter $<3 \mathrm{~nm}$ [19].

Er ions in a solid matrix, such as $\mathrm{Si}$ or $\mathrm{SiO}_{2}$, are $\mathrm{Er}^{3+}$ with an electronic configuration [Xe] $4 \mathrm{f}^{11}$, which generates magnetic and luminescent properties. In fact, after exciting the ions, the radiative transition occurs between the quadruplet $\mathrm{I}_{13 / 2}$ and $\mathrm{I}_{15 / 2}$ with the emission of photons at $1.54 \mu \mathrm{m}$. Si-ncs have shown a good capability of sensitizing [20] the rare-earths that can be excited more efficiently than in pure silicon dioxide. As explained in Section 1, the optical pumping of a material made by a matrix of Er-doped silica where Si-ncs are dispersed, induces an efficient photon absorption by Si ncs where confined excitons are generated. The recombination energy of excitons can be transferred to Er ions and the relaxation of exciting $\mathrm{Er}^{3+}$ generates a radiation at $1.54 \mu \mathrm{m}(\sim 0.8 \mathrm{eV})$.

It is worth noting that the photoluminescence of Si-ncs sensitized Er ions is stable with the temperature. In [21] the temperature dependence of the photoluminescence signal emitted at $1.54 \mu \mathrm{m}$ by the excited Er ions in presence of Si-nc is reported. It is compared to that one of Er-doped crystalline silicon and to Er-doped silica. In particular, the photoluminescence intensity at $1.54 \mu \mathrm{m}$ in case of Er-doped Si-nc is almost independent on the temperature because it decreases of a factor 3 from $20 \mathrm{~K}$ to room temperature. Moreover, at room temperature the signal is two order of magnitude larger than that of $\mathrm{SiO}_{2}$ doped by Er. The photoluminescence from Er-implanted crystalline $\mathrm{Si}$ is almost completely quenched at room temperature, showing the inefficiency of the material system Er:Si at room temperature.

Optical gain of Si-ncs [22] and Er-doped Si-ncs [23] has been demonstrated. In Er-doped ncs a value of $4 \mathrm{~dB} / \mathrm{cm}$ has been achieved [23].

\section{MODELLING AND DESIGN OF Si-nC DBR LASER SOURCE}

In this Section we describe the modelling and design of the $\mathrm{Er}: \mathrm{Si}-\mathrm{nc} / \mathrm{SiO}_{2} \mathrm{rib}$ waveguide, of the laser cavity and of the Bragg mirrors.

\subsection{Er:Si-nc/SiO 2 rib waveguide}

The refractive index of Si-ncs incorporated in a $\mathrm{SiO}_{2}$ matrix has been evaluated by using the Maxwell-Garnett theory [24], allowing to derive the effective dielectric constant by using the following formula:

$$
\frac{\epsilon_{e f f}-\epsilon_{a}}{\epsilon_{e f f}+2 \epsilon_{a}}=\phi \frac{\epsilon-\epsilon_{a}}{\epsilon+2 \epsilon_{a}},
$$

with $\epsilon_{a}$ dielectric constant of the matrix material, $\epsilon$ dielectric constant of the incorporated material depending on the frequency, and $\phi$ the fraction of volume containing the incorporated material. For the system material Si-nc $/ \mathrm{SiO}_{2}, \phi$ corresponds to the concentration of atoms of $\mathrm{Si}$ in excess. At 1.54 $\mu \mathrm{m}$ it results $\epsilon_{S i}=12.1$ and $\epsilon_{S_{i O}}=2.1$, therefore for a $\mathrm{Si}-\mathrm{nc} / \mathrm{SiO}_{2}$ concentration of $1 \%$, we obtain $\epsilon_{\text {eff }}=2.13$, and $n_{\text {eff }}=1.459$. In Table 1 we report the refractive index of the materials we have considered.

Single-mode condition in a rib waveguide can be achieved also with waveguide having relatively large size (core diameter $5 \div 10 \mu \mathrm{m}$ ), particularly useful for obtaining an efficient coupling to the optical fibre. For this reason we have chosen such kind of waveguide. The configuration of the waveguide we have designed is in Figure 1. The electromagnetic analysis of this waveguide has been carried out by using the effective index method (EIM) and the finite element method (FEM).

In Figure 2 the curve limiting the single mode condition is illustrated. In this figure the ratio $\mathrm{w} / \mathrm{H}$ is reported as a function 


\begin{tabular}{|l|l|l|l|l|}
\hline$@ 1.54 \mu \mathrm{m}$ & $\mathrm{Si}$ & $\mathrm{SiO}_{2}$ & $1 \% \mathrm{Si}-\mathrm{nc} / \mathrm{SiO}_{2}$ & $\mathrm{Er}^{3+}: 1 \% \mathrm{Si}-\mathrm{nc} / \mathrm{SiO}_{2}$ \\
\hline Refractive index $n$ & 3.48 & 1.444 & 1.459 & 1.464 \\
\hline Dielectric constant $\epsilon_{r}$ & 12.11 & 2.09 & 2.13 & 2.14 \\
\hline
\end{tabular}

TABLE 1 Refractive index and dielectric constant of studied materials @ $1.54 \mu \mathrm{m}$.

of $\mathrm{h} / \mathrm{H}$, as described by the following equation, derived by the EIM:

$$
\frac{w}{H}=0.1+\frac{h / H}{\sqrt{1.2-(h / H)^{2}}} .
$$

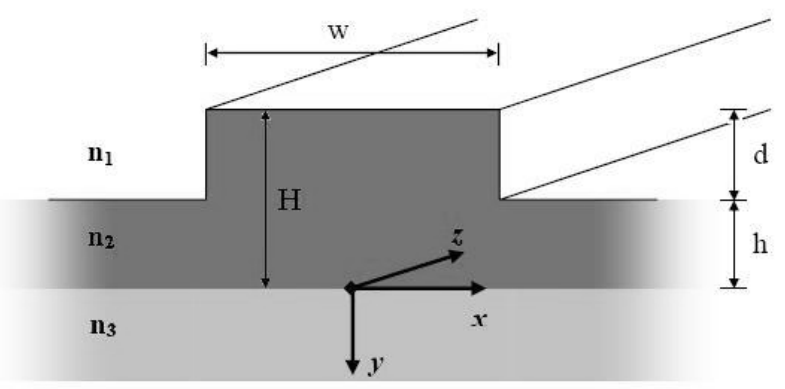

FIG. 1 Geometry of the rib waveguide.

Below the blue curve we can notice only single mode operation, while over that curve multimode operation can be observed mostly.

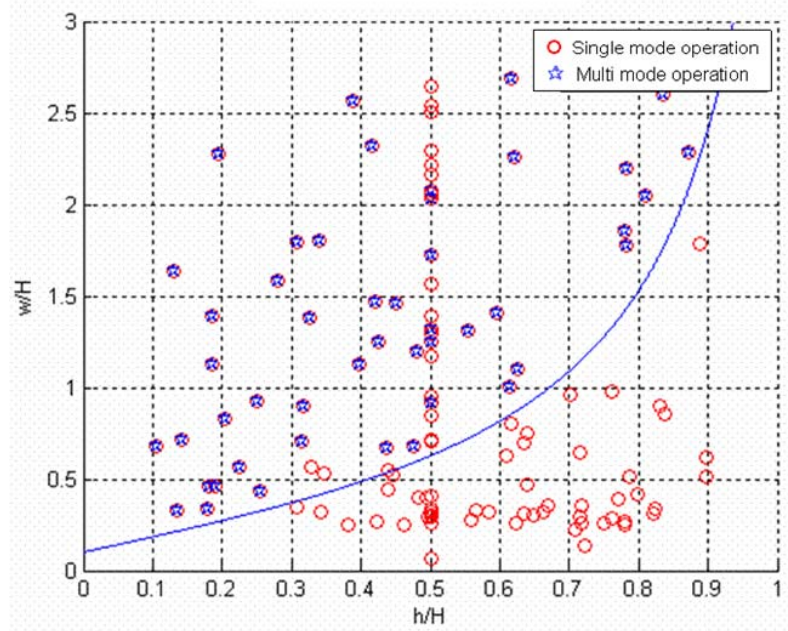

FIG. 2 Dispersion curve of a rib formed by air/Er-doped Si-nc-SiO $/ \mathrm{SiO}_{2}$ for HE modes.

However, in our calculations, a more accurate single mode condition has been used as proposed in [25]

$$
\frac{w}{H} \leq 0.3+\frac{h / H}{\sqrt{1-(h / H)^{2}}} \text { for } 0.5 \leq h / H \leq 1,
$$

which is particularly useful with high $\mathrm{h} / \mathrm{H}$ ratios.

As it is well known, EIM is a semi-analytical, very fast, sufficiently accurate approach, but it is also an efficient technique only after defining the geometry of the waveguiding structure. For this reason we have used also the FEM, because vectorial approaches can be utilized for any kind of waveguide configuration.

Assuming a core of Si-ncs incorporated in an $\mathrm{SiO}_{2}$ material matrix and Er-doped $\left(n_{2}=1.464 @ 1.54 \mu \mathrm{m}\right)$, an air cladding $\left(n_{1}=1 @ 1.54 \mu \mathrm{m}\right)$ and an $\mathrm{SiO}_{2}$ substrate $\left(n_{3}=1.444 @ 1.54\right.$ $\mu \mathrm{m})$, according to Eq. (7) we have determined $H=5 \mu \mathrm{m}$ and $h=3 \mu \mathrm{m}$. Single-mode condition leads to:

$$
\frac{w}{H} \leq 0.3+\frac{0.6}{\sqrt{1-0.6^{2}}}=1.05 \text { for } \quad 0.5 \leq h / H=0.6 \leq 1 \text {. }
$$

Therefore, single mode condition is satisfied by putting $w=$ $0.8, H=4 \mu \mathrm{m}$.

In our case, 2D simulation is enough to detect also higher order modes, if there exist, because the mode analysis allows to investigate all the hybrid modes in the structure. Results we have obtained by using the FEM confirmed the single mode operation or each wave polarization. In fact we have found one quasi-TE mode with $n_{e f f}=1.4547$ and one quasi-TM mode with $n_{\text {eff }}=1.4544$.

3D simulation would be necessary to evaluate the losses in the waveguide. However, we have not yet attempted that evaluation because it is needed to better define the material characteristics, which are related to the fabrication process due to the presence of the Si-ncs.

\subsection{Laser cavity}

Electrical pumping of the active region has been demonstrated to be inadequate with respect to the optical pumping, because a degradation of the performance has been observed with the temperature. Optical pumping power and wavelength can be estimated only after modelling and designing the active medium and the resonant cavity. Pumping wavelength has to be different from those of absorption band peaks of Er ions, because Er ions must be excited only by Sincs, and it should correspond to a low reflectivity region of the material, rather below the stop-band. The choice is restricted to an Ar laser (477 nm), Ar-Kr laser (488 nm) or to a LED at $470 \mathrm{~nm}$. Of course, to avoid further reduction of the gain efficiency a pumping laser would be recommended (we have considered the Ar-Kr laser). As for the operating wavelength of the device we refer to $1.5 \mu \mathrm{m}$, because silicon is transparent, thus allowing integration of all silicon devices, and that it is the wavelength of the telecommunication systems.

The length of the cavity is given by

$$
L=m \frac{c}{2 n v_{m}}=m \frac{\lambda_{0}}{2 n}=m \frac{\lambda_{g}}{2}
$$


where $m$ is an integer, $n$ the refractive index of the active medium, $\lambda_{0}$ the free space wavelength and $\lambda_{g}=\lambda_{0} / n$ the wavelength in the waveguide, and $v_{m}$ the frequency.

In our case, by taking into account that the gain is quite low ( $\leq 4 \mathrm{~dB} / \mathrm{cm}$ ) the length of the cavity obtained from Eq. (9) results $2.63 \mathrm{~cm}$, and it is large enough with respect to the gain value.

The waveguide we have designed is depicted in Figure 3 [23]. With a Si concentration of $34 \%$ and an Er concentration of $0.03 \%$, as in [23], a gain value of $3.7 \mathrm{~dB} / \mathrm{cm}$ around $\lambda=1.537$ $\mu \mathrm{m}$ has been obtained experimentally for a cavity length of $9 \mathrm{~mm}$, by using a pumping power and wavelength of 1.5 $\mathrm{Wcm}^{-2}$ and $477 \mathrm{~nm}$, respectively.

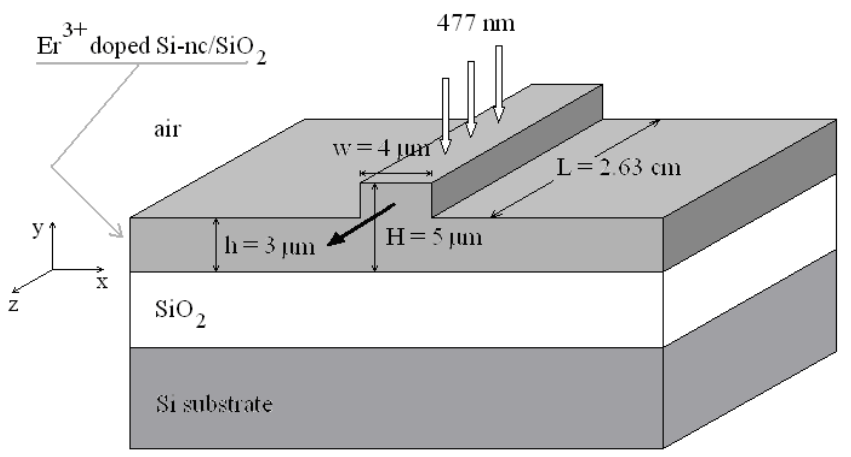

FIC. 3 Er-doped Si-nc rib waveguide.

Assuming mirror reflectivities $R_{1}=100 \%$ and $R_{2}=97 \%$, the cavity gain results in the $3-4 \mathrm{~dB} / \mathrm{cm}$ range, when the cavity loss is of the order of $1 \mathrm{dBcm}^{-1}$.

In conclusion, a gain value higher than the threshold one can be achieved by appropriately designing the material, the pump characteristics, the cavity length and the mirror's reflectivity.

As it can be seen, the Er:Si-nc laser designed is power consuming and uncompetitive with respect to the III-V semiconductor lasers. Much effort needs to improve these laser sources, which are very useful because of their compatibility with electronic integrated circuits and low cost. Electrical pumping would be an important advance due to its low cost and good efficiency.

\subsection{Bragg mirrors}

Using a stack of $\mathrm{Si}$ and $\mathrm{SiO}_{2}$ layers Bragg mirrors can be tailored at a specific wavelength. Since the refractive index of the active medium is low $\left(n_{2}=1.464\right)$, cleaved facets technique cannot be used to fabricate the mirrors due to high losses and low reflectivity ( $R=3.5 \%$ ). Distributed Bragg Reflector configuration is particularly suitable due to the compatibility of materials involved in.

With reference to Figure 4, we observe that the Bragg wavelength, for $\theta=90^{\circ}$, is given by $\lambda_{g, B}=2 n \Lambda$, where $n$ is the refractive index $\left(n=n_{\text {high }}, n_{\text {low }}\right)$ and $\Lambda$ is the grating period, by considering only the first diffraction order $(m=1)$.

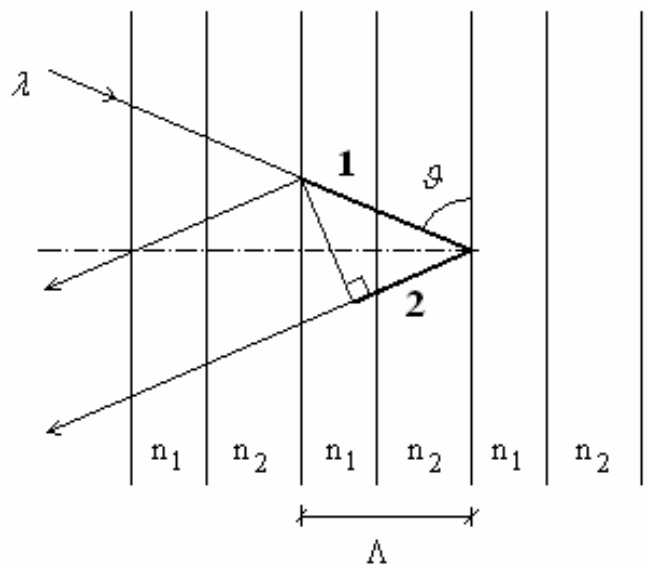

FIG. 4 Optical path difference between two waves reflected by the grating.

From this figure the optical path difference $\Delta p$ has been calculated:

$$
\begin{aligned}
\Delta p & =\overline{1}+\overline{2}=\frac{\Lambda}{\sin \theta}+\frac{\Lambda \cos \left(180^{\circ}-2 \theta\right)}{\sin \theta} \\
& =\frac{\Lambda}{\sin \theta}(1-\cos 2 \theta)=2 \Lambda \sin \theta=m \lambda .
\end{aligned}
$$

If the grating period is multiple of half wavelength, we can derive the thickness of each layer as a quarter of the waveguide wavelength:

$$
\Lambda=\frac{\lambda_{0}}{2 n} \Rightarrow d_{1,2}=\frac{\lambda_{0}}{4 n} .
$$

Therefore, we have obtained

$$
\begin{aligned}
& \mathrm{Si}: d_{1}=\lambda_{0} /\left(4 n_{1}\right)=110_{n} m\left(n_{1}=3.48\right) \\
& \mathrm{SiO}_{2}: d_{2}=\lambda_{0} /\left(4 n_{2}\right)=266_{n} m\left(n_{2}=1.44\right) .
\end{aligned}
$$

The reflectance now assumes the following expression:

$$
R=\left[\frac{1-\frac{n_{T}}{n_{0}}\left(\frac{n_{H}}{n_{L}}\right)^{2 p}}{1+\frac{n_{T}}{n_{0}}\left(\frac{n_{H}}{n_{L}}\right)^{2 p}}\right]^{2} \approx \tanh ^{2}\left[2 p \frac{n_{H}-n_{L}}{n_{H}+n_{L}}\right]
$$

where $Y=n_{T} / n_{0}\left(n_{H} / n_{L}\right)^{2 p}$ is the optical admittance of a stack of $\mathrm{p}$ layer pairs with $n_{0}$ refractive index of the first layer and $n_{T}$ of the last layer.

Calculations of the number of layers of input and output Bragg mirrors resulted in four pairs of layers at the input and three at the output, giving reflectivity values equal to $99.8 \%$ (input mirror) and $97.0 \%$ (output mirror), respectively.

In Figure 5 the top view of the designed laser is shown.

\section{CONCLUSIONS}

In this paper we have described the modelling and design of the optical components of a Si-based DBR laser including a Si-ncs sensitized Er-doped $\mathrm{SiO}_{2}$ rib waveguide, to be used 


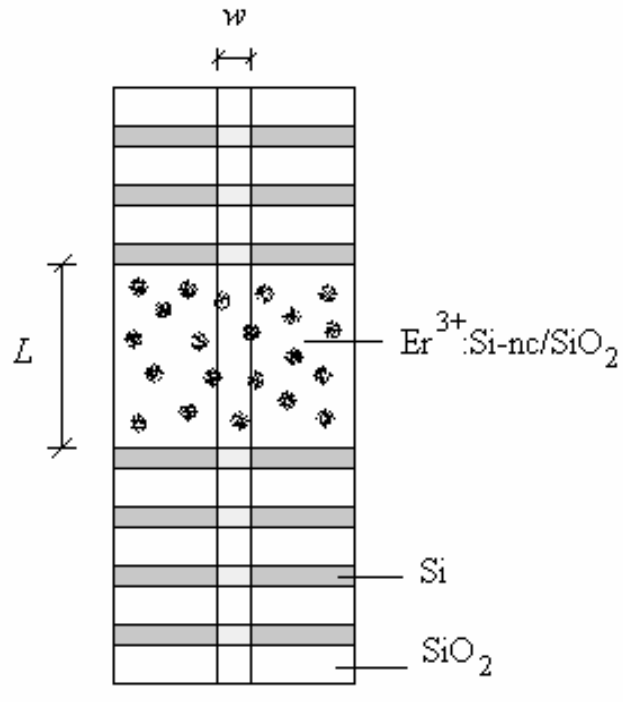

FIC. 5 Top view of the Si-nc DBR laser.

in telecommunication systems at operating wavelengths in the C-band. In particular, the structure and optical properties of the silicon nanocrystals are described, and the modelling and design of the Er:Si-nc/ $\mathrm{SiO}_{2}$ rib waveguide, of the optical cavity and of the Bragg gratings are reported. Two different numerical methods have been used for modelling the waveguide, i.e. the effective index method and the finite element method. Calculations of the monomode conditions of the waveguide have been carried out, according to [25] and [26]. We have also evaluated the mode confinement, by using both the Effective Index Method and the Finite Element Method.

\section{References}

[1] M.A. Green, J. Zhao, A. Wang, P.J. Reece and M. Gal, "Efficient silicon light-emitting diodes" Nature 412, 805-808 2001.

[2] W.L. Ng, M.A. Lourenco, R.M. Gwilliam, S. Ledian, G. Shao and K.P. Homewood, "An efficient room-temperature silicon-based lightemitting diode"Nature 410, 192-194 2001.

[3] G. Franzò, S. Coffa, F. Priolo and C. Spinella, "Mechanism and performance of forward and reverse bias electroluminescence at $1.54 \mu \mathrm{m}$ from Er-doped Si diodes" J. Appl. Phys. 81, 2784-2793 1997.

[4] A.G. Cullis, L.T. Canham, and P.D.J. Calcott, "The structural and luminescence properties of porous silicon" Appl. Phys. Rev. 82, 909-965 1997.

[5] 0. Bisi, S. Ossicini and L. Pavesi, "Porous silicon: a quantum sponge structure for silicon based optoelectronic" Surf. Sci. Rep. 264, 1-1262000.

[6] A. Irrera, D. Pacifici, M. Miritello, G. Franzò, F. Priolo, F. Iacona, D. Sanfilippo, G. Di Stefano and P.G. Fallica, "Electro-luminescence properties of light emitting devices based on silicon nanocrystals" Physica E 16, 395-399 2003.
[7] L. Pavesi, L. Dal Negro, G. Mazzoleni, G. Franzò and F. Priolo, “Optical gain in silicon nano-crystals" Nature 408, 440-444 2000.

[8] G. Franzò, D. Pacifici, V. Vinciguerra, F. Priolo and F. lacona, “ $\mathrm{Er}^{3+}$ ions-Si nanocrystals interactions and their effects on the luminescence properties" Appl. Phys. Lett. 76, 2167-2169 2000.

[9] W. H. Chang, A. T. Chou, W. Y. Chen, H. S. Chang, T. M. Hsu, Z. Pei, P. S. Chen, S. W. Lee, S. Lai, S. C. Lu and M-J. Tsai, "Room temperature electroluminescence at 1.3 and $1.5 \mu \mathrm{m}$ from $\mathrm{Ce} / \mathrm{Si}$ self assembled quantum dots" Appl. Phys. Lett. 83, 2958-2960 2003.

[10] F. Lucarz and A.J. Kenyon, "Silicon nanocrystals in erbium-doped silica for optical amplifiers" London Communications Symposium, 2003.

[11] K. Imakita, M. Fujii and S. Hayashi, "Spectrally resolved energy transfer from excitons in Si nanocrystals to Er ions" Phys. Rev. B 71, 193301(1-4) 2005.

[12] H. Rong, R. Jones, A. Liu, O. Cohen, D. Hak, A. Fang and M. Paniccia, "A continuous-wave Raman silicon laser" Nature 433, 725-728 2005.

[13] R. B. Hammond and R. N. Silver, "Temperature dependence of the exciton lifetime in high-purity silicon" Appl. Phys. 36, 68-71 1980.

[14] M. A. Tamor and J. P. Wolfe, "Drift and Diffusion of Free Excitons in Si" Phys. Rev. Lett. 44, 1703-1706 1980.

[15] R. N. Hall, “Electron-Hole Recombination in Germanium" Phys. Rev. 87, 3871952.

[16] W. Shockley and W. T Read, "Statistics of the Recombinations of Holes and Electrons" Phys. Rev. 87, 835-842 1952.

[17] J. D. Cuthbert, "Recombination Kinetics of Excitonic Molecules and Free Excitons in Intrinsic Silicon" Phys. Rev. B 1, 1552-1557 1970.

[18] F. Iacona, G. Franzò and C. Spinella, "Correlation between luminescence and structural properties of Si Nanocrystals" J. Appl. Phys. 87, 1295-1303 2000.

[19] M. V. Wolkin, J. Jorne, P. M. Fauchet, G. Allan and C. Delerue, "Electronic States and Luminescence in Porous Silicon Quantum Dots: The Role of Oxygen" Phys. Rev. Lett. 82, 197-200 1999.

[20] . Lee, J. H. Shin and N. Park, “Optical Gain at $1.5 \mathrm{~mm}$ in Nanocrystal Si-Sensitized Er-Doped Silica Waveguide Using Top-Pumping 470 nm LEDs" J. Lightwave Technol. 23, 19-25 2005.

[21] A. Irrera, "Light emitting devices based on silicon nanostructures" Ph. D. Thesis, 2004.

[22] K. L. Shaklee, R. E. Nahaori and L. F. Leheny, "Optical gain in semiconductors" J. Lumin. 7, 284-309 1973.

[23] H. S. Han, S. Y. Seo and J. H. Shin, "Optical gain at $1.54 \mu \mathrm{m}$ in erbium-doped silicon nanocluster sensitized waveguide" Appl. Phys. Lett. 79, 4568-4570 2001.

[24] D. Bedeaux and J. Vlieger, Optical Properties of Surfaces (Imperial College Press, 2004).

[25] R. A. Soref, J. Schmidtchen and K. Petermann, "Large Single-Mode Rib Waveguides in CeSi-Si and $\mathrm{Si}-\mathrm{on}-\mathrm{SiO}_{2}$ " IEEE J. Quantum Elect. 27, 1971-1974 1991.

[26] S. P. Pogossian, L. Vescan and A. Vonsovici, "The Single-Mode Condition for Semiconductor Rib Waveguides with Large Cross Section" J. Lightwave Technol. 16, 1851-1853 1998. 